\title{
Microbiome-derived metabolome as a potential predictor of response to cancer immunotherapy
}

\author{
Agnieszka Beata Malczewski (D) , ,2,3 Severine Navarro,, ${ }^{4,5}$ Jermaine IG Coward, , ${ }^{1,2}$ \\ Natkunam Ketheesan ${ }^{3}$
}

To cite: Malczewski AB Navarro S, Coward JIG, et al. Microbiome-derived metabolome as a potential predictor of response to cancer immunotherapy. Journal for ImmunoTherapy of Cancer 2020;8:e001383. doi:10.1136/ jitc-2020-001383

Accepted 10 September 2020

Check for updates

(C) Author(s) (or their employer(s)) 2020. Re-use permitted under CC BY-NC. No commercial re-use. See rights and permissions. Published by BMJ.

${ }^{1}$ Icon Cancer Centre, Wesley and South Brisbane, Brisbane, Queensland, Australia

${ }^{2}$ Faculty of Medicine, University of Queensland, Brisbane,

Queensland, Australia

${ }^{3}$ Science and Technology, University of New England, Armidale, New South Wales, Australia

${ }^{4}$ Department of Immunology, QIMR Berghofer Medical

Research Institute, Brisbane,

Queensland, Australia

${ }^{5}$ Woolworths Centre for

Childhood Nutrition Research,

Faculty of Health, Queensland

University of Technology,

Brisbane, Queensland, Australia

\section{Correspondence to}

Dr Agnieszka Beata Malczewski; agnieszka.malczewski@icon. team

\begin{abstract}
Cancer immunotherapy with checkpoint blockade has become standard of care treatment for numerous cancer types. Despite this, robust predictive biomarkers are lacking. There is increasing evidence that the host microbiome is a predictor of immunotherapy response, although the optimal host microbiome has not been defined. Metabolomics is a new area of medicine that aims to analyze the metabolic profile of a biological system. The microbiome-derived metabolome (fecal and serum) represents the end products of microbial metabolism and these may be functionally more important than the distinct bacterial species that comprise a favorable microbiome. Short-chain fatty acids (SCFA) are metabolites produced by gut microbiota and have a role in T cell homeostasis, including differentiation of regulatory $T$ cells. Recent studies have confirmed differential expression of SCFA for immunotherapy responders compared with nonresponders. We propose that the microbiome metabolome, with a focus on SCFA may be a novel predictive biomarker for immunotherapy efficacy.
\end{abstract}

Immunotherapy has revolutionized the treatment landscape in medical oncology and has led to improvements in survival across many solid tumor subtypes. Despite this, a significant proportion of cancer patients still do not have a durable response to immunotherapy. Currently available biomarkers are unable to reliably select patients for optimal clinical benefit. Recently, investigators have looked at host-based factors as possible predictors of response to immunotherapy. Multiple studies have confirmed an association between a favorable microbiome and enhanced responses to immunotherapy. Despite this, there has been no consensus over which microbial species enhance response rates to immunotherapy treatment. ${ }^{1}$ The activity of the microbiome is reflected through its metabolic profile or metabolome and can be quantitatively assessed through mass spectrometry-based techniques. Correlation of metabolomic data together with taxonomic composition of the microbiome may enable identification of a metabolic signature that is unique to immunotherapy responders. This metabolic signature could have application as a predictor of response to cancer immunotherapy.

The microbiome consists of the trillions of commensal microbes that live within their human hosts. ${ }^{1}$ The microbiome has been shown to influence immunity and help regulate responses to checkpoint inhibitor therapy. ${ }^{2}$ Clinical trials continue to investigate means of modulating the microbiome to elicit more favorable responses to immunotherapy. ${ }^{1}$ In a landmark study looking at melanoma patients undergoing anti-PD-1 immunotherapy, Gopalakrishnan et al (2018) found significant differences in the microbial composition and diversity of responders, compared with non-responders. ${ }^{2}$ Those patients with a sustained response to treatment had a significantly higher fecal microbiome alpha diversity $(\mathrm{p}<0.01)$ together with a greater abundance of bacteria from the Ruminococcaceae family. ${ }^{2}$ Other investigators have similarly assessed microbiome composition of patients undergoing checkpoint inhibitor therapy. The taxa that have been associated with favorable responses have varied between studies. ${ }^{12}$ Conversely, low microbiome diversity, including recent use of antibiotics prior to initiation of checkpoint inhibitor therapy have been associated with poorer treatment responses, although these findings have not been universal. ${ }^{1}$

Possible techniques to modulate the microbiome include the use of dietary intervention, prebiotics or probiotics, bacteria consortia or fecal transplantation. ${ }^{1}$ Most of these techniques have foreseeable problems in terms of the difficulties associated with reproducing the complex community that constitutes a 'favorable microbiome'. The practicalities and standardization of protocols for techniques such as fecal transplant are challenging and not entirely risk-free. In 
view of this, it may not be realistic to routinely integrate such modalities into daily clinical practice.

Metabolomics is a relatively new field of medicine that investigates the set of metabolites expressed by a particular biological system. Metabolic signatures have already been developed for several chronic disease states, including diabetes, cardiovascular disease and chronic respiratory diseases. ${ }^{3}$ Analysis can be performed on bodily fluids including serum, urine, feces, saliva as well as tumor tissue. Cancer metabolomics is an active and expanding field. In the setting of checkpoint inhibitor therapy, the metabolome could provide insight into the functional microbiome-host interaction and may enable a greater understanding of whether the metabolic activity of bacterial communities is more important than their exact taxonomic constitution.

Commensal microorganisms present in the gut have important roles in regulation of local, innate and adaptive immunity. Microbial metabolic activity in the gut can drive diverse pathological systemic immune responses including autoimmune conditions such as diabetes, rheumatoid arthritis, inflammatory bowel disease and asthma. ${ }^{45}$ For example, in neonates, distinct fecal metabolomes are associated with an excess of pro-inflammatory metabolites, which promote $\mathrm{CD} 4 \mathrm{~T}$ cell dysfunction resulting in childhood atopy and asthma. ${ }^{4}$ A key function of gut bacteria is to convert dietary substrates into metabolites, such as short-chain fatty acids, phenolic compounds, N-nitroso compounds or bile acids. ${ }^{2}$ Short-chain fatty acids (SCFA) have been implicated as key metabolites in the regulation of $\mathrm{T}$ cell homeostasis ${ }^{6}$ (figure 1). Depending on immunological conditions, including presence or absence of key cytokines, SCFA were able to regulate $\mathrm{T}$ cell differentiation into either effector or regulatory (Treg) cells. ${ }^{6}$ The efficacy of checkpoint inhibitor therapy is affected by endocrine, metabolic and environmental conditions. A favorable balance of $\mathrm{T}$ effector cells: Treg cells is required in the tumor microenvironment in order to enhance checkpoint inhibitor efficacy. ${ }^{5}$ Certainly, the immunosuppressive activity of Tregs in the tumor microenvironment is considered detrimental to cancer immunotherapy outcomes. ${ }^{5}$ In keeping with this, Treg depletion has also been put forward as a possible future treatment strategy that could be used in combination with current checkpoint blockade. ${ }^{5}$ Thus, it is possible that the common end metabolic products of diverse microbial communities could shape the immune response in patients on checkpoint inhibitor therapy through effects on $\mathrm{T}$ cell homeostasis or through other as yet undiscovered mechanisms (figure 1). These metabolic products could also be a potential therapeutic target for modulating the immune response.

Studies to date have looked at the emerging role of metabolomic profiling and its association with immunotherapy efficacy. There is increasing evidence that systemic SCFA may modulate checkpoint inhibitor responses in humans. ${ }^{78}$ Nomura et al (2020) published a prospective cohort study of 52 patients with metastatic malignancy who

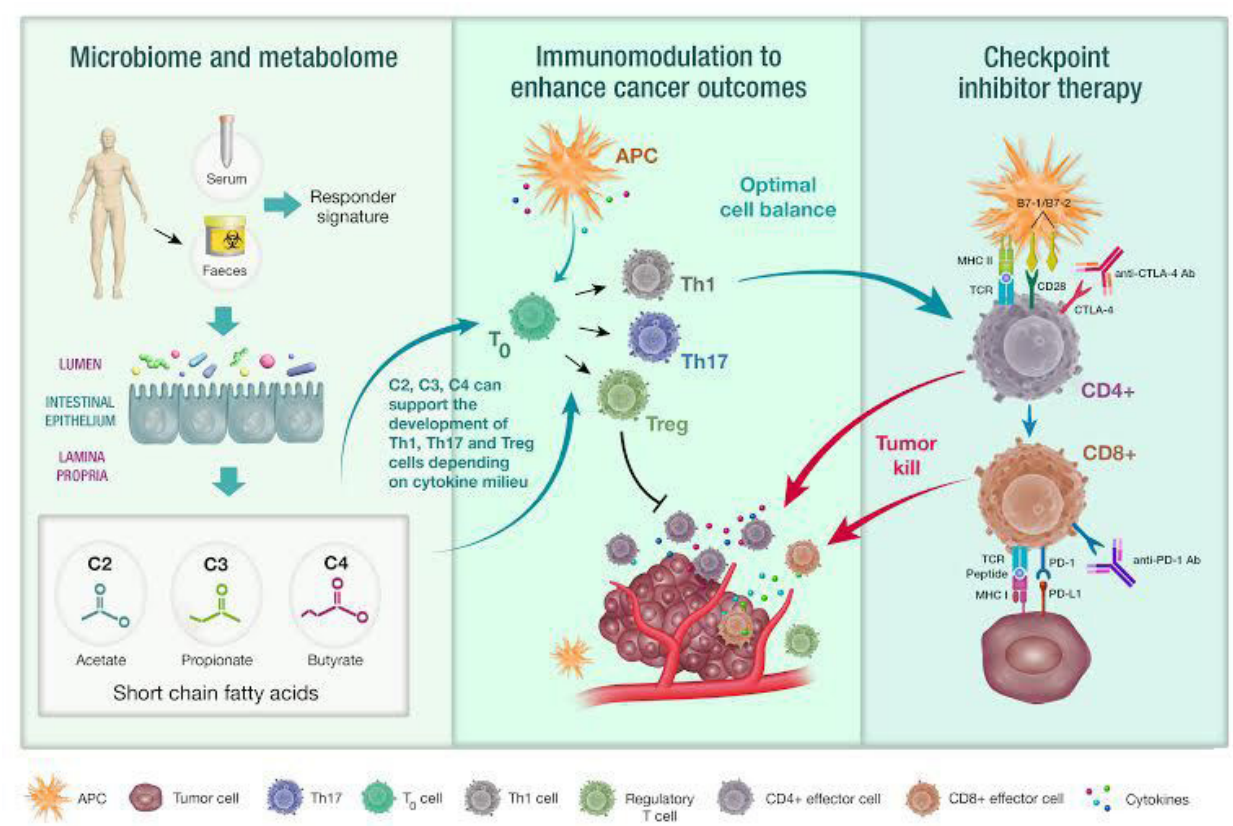

Figure 1 Microbiome-derived metabolome as a predictor of response to cancer immunotherapy. Responses to checkpoint immunotherapy have been associated with a diverse fecal microbiome. Short-chain fatty acids, including acetate (C2), propionate (C3) and butyrate (C4) are products of bacterial fermentation of dietary fiber and are known to induce T cell differentiation (TO). The serum or fecal microbiome-derived metabolome can quantify the metabolic products of microbial communities and could be used as a predictive biomarker for identifying long-term responders to checkpoint immunotherapy (right panel). Short-chain fatty acids can promote both effector and regulatory $T$ cell subsets, depending on the cytokine and immunological milieu (middle and right panel). Optimal conditions for checkpoint inhibitor therapy to facilitate tumor cell killing include increased $\mathrm{T}$ effector cell to Treg cell ratio. 
were treated with nivolumab or pembrolizumab. ${ }^{7}$ Both fecal and plasma concentrations of SCFA were measured for patients, who were categorized as either responders or non-responders. Fecal concentrations of acetic acid, propionic acid, butyric acid and valeric acid and plasma concentrations of propionic acid and isovaleric acid were significantly elevated for responders, compared with non-responders $(\mathrm{p}<0.05){ }^{7}$ Similarly, Botticelli et al (2020) assessed the fecal microbiome metabolome for 11 patients with non-small cell lung cancer undergoing treatment with nivolumab. Investigators found that patients with early disease progression (defined as disease progression within 3 months of starting nivolumab) had a microbiome metabolome that was characterized by low levels of both SCFA (propionic, butyric, acetic and valeric acids) and amino acids (lysine, isoleucine and glutamic) but high levels of alkanes, methyl-ketones and $p$-cresol. ${ }^{8}$

In contrast to this, a recently published study of $(\mathrm{n}=85)$ metastatic melanoma patients receiving antiCTLA-4 therapy confirmed that low baseline butyrate and low baseline propionate (serum) were associated with increased progression free survival $(p=0.0015$ and $\mathrm{p}=0.0029$, respectively).$^{9}$ As these patients were receiving single agent treatment, further studies are required to investigate the effects of SCFA on treatment outcomes in patients receiving combination immunotherapy. Mouse studies suggested that butyrate reduced the efficacy of CTLA-4 blockade by limiting CTLA-4 induced dendritic cell maturation together with decreased ICOS expression on T cells and reduced accumulation of memory T cells. ${ }^{9}$ Together, these recent studies suggest that SCFA are key regulators of systemic immunity during checkpoint inhibitor therapy and may be surrogate markers of specific microbial communities.

Apart from utility as a predictive biomarker, identifying and correcting certain metabolic derangements could potentially be used to help re-sensitize patients who may be identified as resistant to immunotherapy or who have developed progressive disease. The therapeutic effect of SCFA supplementation is currently under study in the setting of inflammatory bowel disease, where dysbiosis is prominent. ${ }^{10}$ To date, administration of SCFA either through oral administration or through the use of enemas has not had consistent clinical results. ${ }^{10}$

Integration of metabolomics and gut microbiota profiling is an excellent and obvious target for future use as both a predictive biomarker and as a point for manipulating the host immune response. Host related factors have long been overlooked in favor of tumor-related factors in oncology, which is a significant oversight. SCFA are known to be key regulators of immune function and appear to be expressed differentially between immunotherapy responders and non-responders. We hypothesize that the serial evaluation of the microbiome-derived metabolome in a large cohort of patients on immunotherapy will enable the identification of a unique SCFA metabolic signature that may be robust enough to act as predictive biomarker for immunotherapy efficacy. Furthermore, this may become a therapeutic target for enhancing responses to checkpoint inhibitor therapy.

Twitter Severine Navarro @NavarroSeverine

Acknowledgements We wish to acknowledge medical illustrator, Madeleine Kersting Flynn, QIMR Berghofer Medical Research Institute.

Contributors $A B M$ had the original concept for this manuscript. All authors have had an equal contribution in writing the manuscript.

Funding The authors have not declared a specific grant for this research from any funding agency in the public, commercial or not-for-profit sectors.

Competing interests None declared.

Patient consent for publication Not required.

Provenance and peer review Not commissioned; externally peer reviewed.

Open access This is an open access article distributed in accordance with the Creative Commons Attribution Non Commercial (CC BY-NC 4.0) license, which permits others to distribute, remix, adapt, build upon this work non-commercially, and license their derivative works on different terms, provided the original work is properly cited, appropriate credit is given, any changes made indicated, and the use is non-commercial. See http://creativecommons.org/licenses/by-nc/4.0/.

\section{ORCID iD}

Agnieszka Beata Malczewski http://orcid.org/0000-0002-9110-0060

\section{REFERENCES}

1 McQuade JL, Daniel CR, Helmink BA, et al. Modulating the microbiome to improve therapeutic response in cancer. Lancet Oncol 2019;20:e77-91.

2 Gopalakrishnan V, Spencer CN, Nezi L, et al. Gut microbiome modulates response to anti-PD-1 immunotherapy in melanoma patients. Science 2018;359:97-103.

3 Mastrangelo A, Barbas C. Chronic Diseases and Lifestyle Biomarkers Identification by Metabolomics. In: Sussulini A, ed. Metabolomics: from Fundamentals to clinical applications. advances in experimental medicine and biology. 965. Springer, Cham, 2017.

4 Fujimura KE, Sitarik AR, Havstad S, et al. Neonatal gut microbiota associates with childhood multisensitized atopy and $\mathrm{T}$ cell differentiation. Nat Med 2016;22:1187-91.

5 Pitt JM, Vétizou M, Daillère R, et al. Resistance mechanisms to Immune-Checkpoint blockade in cancer: tumor-intrinsic and -Extrinsic factors. Immunity 2016;44:1255-69.

6 Park J, Kim M, Kang SG, et al. Short-Chain fatty acids induce both effector and regulatory $\mathrm{T}$ cells by suppression of histone deacetylases and regulation of the mTOR-s6K pathway. Mucosal Immunol 2015;8:80-93.

7 Nomura M, Nagatomo R, Doi K, et al. Association of short-chain fatty acids in the gut microbiome with clinical response to treatment with nivolumab or pembrolizumab in patients with solid cancer tumors. JAMA Netw Open 2020;3:e202895.

8 Botticelli A, Vernocchi P, Marini F, et al. Gut metabolomics profiling of non-small cell lung cancer (NSCLC) patients under immunotherapy treatment. J Transl Med 2020;18:49.

9 Coutzac C, Jouniaux J-M, Paci A, et al. Systemic short chain fatty acids limit antitumor effect of CTLA-4 blockade in hosts with cancer. Nat Commun 2020;11:2168.

10 Russo E, Giudici F, Fiorindi C, et al. Immunomodulating activity and therapeutic effects of short chain fatty acids and tryptophan postbiotics in inflammatory bowel disease. Front Immunol 2019;10:2754. 